\title{
TESTING COMMON ENVELOPE THEORIES WITH WHITE DWARF BINARIES
}

\author{
G. Nelemans ${ }^{1}$ and C.A. Tout ${ }^{1}$
}

RESUMEN

Determinamos las posibles masas y radios de las progenitoras de enanas blancas en binarias a partir de ajuste. a modelos evolutivos estelares detallados y los utilizamos para reconstruir la fase de transferencia de masa durante la cual se formó la enana blanca. Confirmamos lo encontrado anteriormente: que en la primera fase de transferencia de masa, en la evolución binaria que condujo a la formación de un par cercano de enanas blancas. el formalismo estándar de una envolvente común que se basa en el equilibrio de energía simplemente no se aplica. Lo que puede explicar las observaciones es un formalismo con base en equilibrio del momento angular. Nuestro análisis se extendió a todas las binarias cercanas en las que al menos una componente es enana blanca. Al comparar los dos mostramos que el formalismo con base en el equilibrio del momento angular puede explicar todas las observaciones por lo menos tan bien como el formalismo de energía.

\section{ABSTRACT}

We determine the possible masses and radii of the progenitors of white dwarfs in binaries from fits to detailed stellar evolution models and use these to reconstruct the mass-transfer phase in which the white dwarf was formed. We confirm the earlier finding that in the first phase of mass transfer in the binary evolution leading to a close pair of white dwarfs, the standard common-envelope formalism based on energy balance in the system. does not work. A formalism based on angular momentum balance can explain the observations. We extend our analysis to all close binaries with at least one white dwarf component. Comparing the two. we show that the formalism based on angular momentum balance can explain all observations at least as well as the energy formalism

Key Words: BINARIES: CLOSE - WHITE DWARFS

\section{INTRODUCTION}

Now that double white dwarfs are discovered regularly it has become more and more clear that most of them have a mass ratio close to unity (e.g. Maxted \& Marsh 1999; Maxted, Marsh, \& Moran 2002). This is contrary to what is expected from standard population synthesis calculations (e.g. Iben, Tutukov, \& Yungelson 1997; Han 1998). A possible resolution of this issue was investigated by Nelemans, Verbunt, Yungelson, \& Portegies Zwart (2000); Nelemans, Yungelson, Portegies Zwart, \& Verbunt (2001). In these papers the observed masses of three double white dwarfs and the well known core-mass - radius relation were used to reconstruct the evolution of the binary back to the two mainsequence stars. It followed that the first phase of mass transfer could not be described by the standard common-envelope formalism based on energy balance. Instead a formalism based on the angular momentum balance was proposed that could explain the observed systems.

\footnotetext{
${ }^{1}$ Instite of Astronomy, University of Cambridge, Cambridge, UK
}

Since then, quite a few more double white dwarfs have been discovered. In particular the SPY project (Napiwotzki, Christlieb, Drechsel, Hagen. Heber, Homeier, Karl, Koester, Leibundgut. Marsh. Moehler, Nelemans, Pauli, Reimers, Renzini, \& Yungelson 2001), a large survey on the ESO Very Large Telescope, to measure radial velocity variations of some thousand white dwarfs in order to detect duplicity, has, and will enlarge the known double white dwarf sample. We therefore repeat the analysis of Nelemans et al. (2000), including the new discoveries.

\section{RESULTS}

For details on the method and observational dat it used here we refer to Nelemans \& Tout (2003). In the following we infer the values of the free parameters a $\lambda$ and $\gamma$ in the common-envelope formalisms bilsed on energy:

$$
\frac{G M_{\mathrm{g}} M_{\mathrm{e}}}{\lambda R_{\mathrm{g}}}=\alpha\left(\frac{G \cdot M_{\mathrm{c}} m}{2 a_{\mathrm{f}}}-\frac{G . I_{\mathrm{g}} m}{2 a_{\mathrm{i}}}\right)
$$



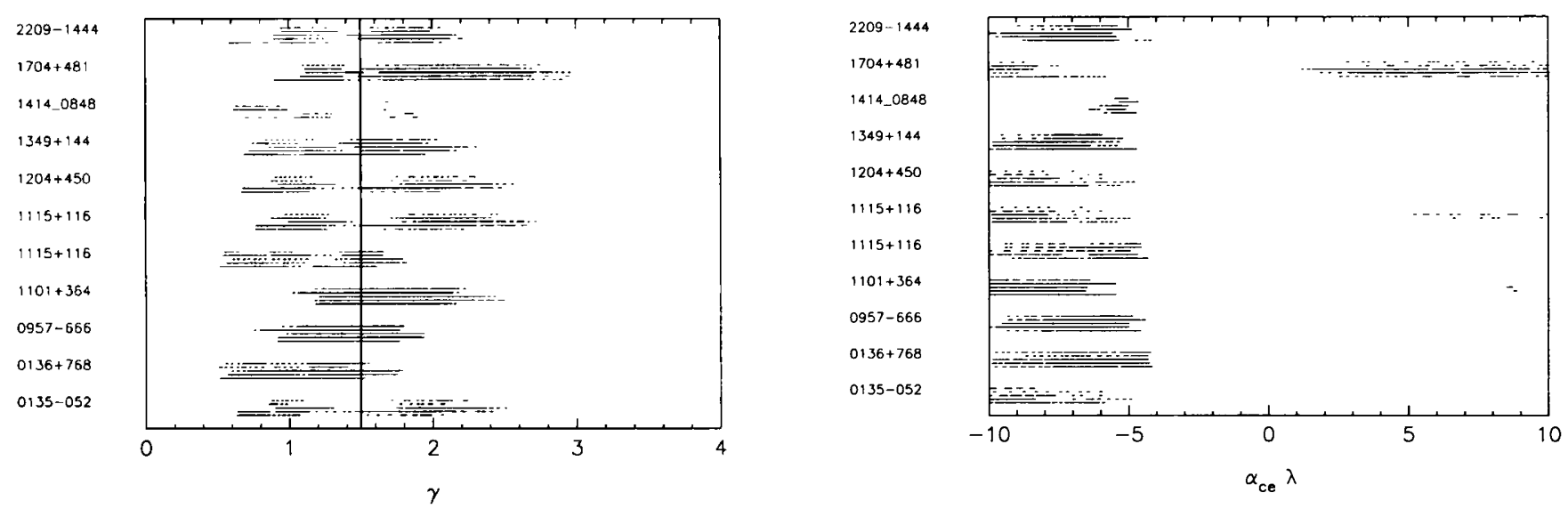

Fig. 1. Left: Reconstructed $\gamma$ values for the first phase of mass transfer in the formation of double white dwarfs.Right: reconstructed $\alpha \lambda$ values for the sarne. The horizontal lines are made up of small dashes representing the reconstructed values of $\gamma$ and $\alpha \lambda$ for different values of the mass of the progenitor of the white dwarf and the companion. The different lines for each object represent different values of the white dwarf mass (within $0.05 \mathrm{M}_{\odot}$ ).

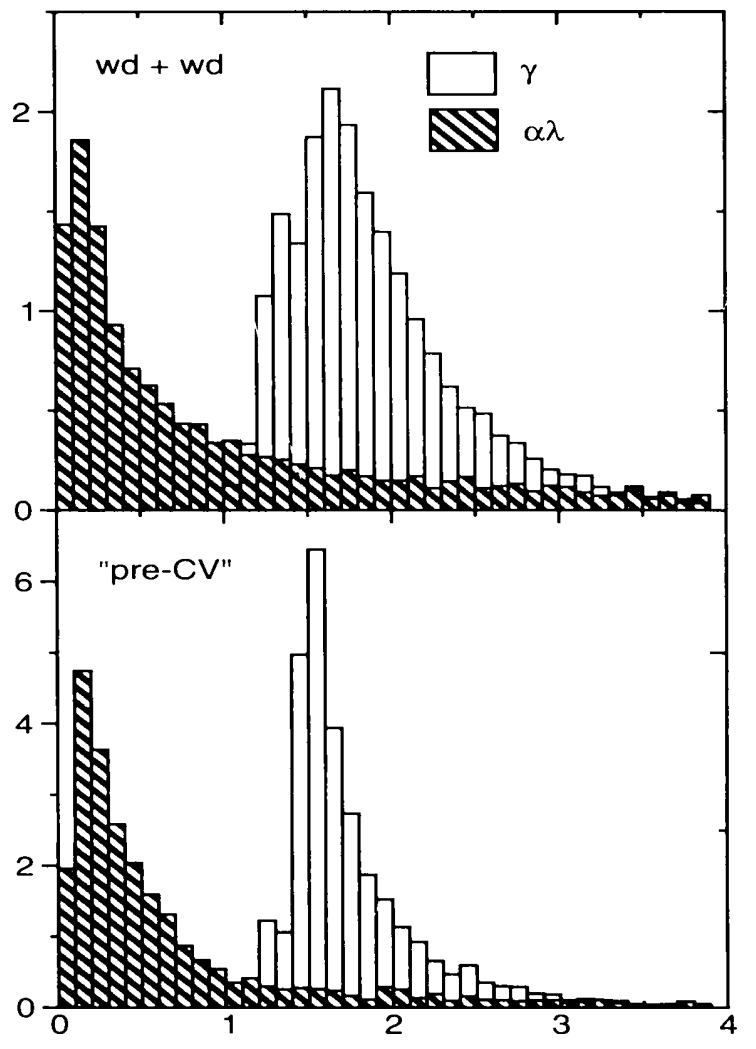

Fig. 2. Histograns of the reconstructed $\gamma$ and $\alpha \lambda$ values for the last phase of mass transfer leading to the observed double white dwarf binaries (top panel) and for the mass transfer leading to the observed pre-CV's and related objects.

where subscripts g, e and c are for giant, envelope and core respectively and angular momentum:

$$
\frac{\Delta J}{J}=\gamma \frac{\Delta M}{M+m} .
$$

In Fig. 1 we show the reconstructed values of $\gamma$ and $\alpha \lambda$ for the energy formalism for the reconstruction of the first phase of mass transfer in the progen- itors of double white dwarfs. We confirm the finding of Nelemans et al. (2000) that this phase cannot be described with the standard common-envelope formalism, as the efficiency has to be negative (expect for WD 1704+481). We used the same method to reconstruct the last mass transfer phase leading to the observed double white dwarfs. A histogram of the reconstructed $\gamma$ and $\alpha \lambda$ values if shown in Fig. 2 (top panel). We also collected the paramters of more than 30 observed pre- $\mathrm{CV}$ and related binaries (binaries with a white dwarf and a main-sequence companion) and used the reconstruction method for these binaries. The results are shown in the bottom panel of Fig. 2. The conclusion for these two mass transfer phases is that both the standard formalism and the angular momentum formalism can explain the observations. For the energy formalism we find quite low values for the efficiency, while for the angular momentum formalism we find values of $\gamma$ clustered around 1.5 .

\section{REFERENCES}

Han, Z. 1998, MNRAS, 296, 1019

Iben, Jr, I., Tutukov, A. V., \& Yungelson, L. R. 1997, ApJ, 475, 291

Maxted, P. F. L. \& Marsh, T. R. 1999, MNRAS, 307, 122

Maxted, P. F. L., Marsh, T. R., \& Moran, C. K. J. 2002, MNRAS, 332, 745

Napiwotzki, R., Christlieb, N., Drechsel, H., et al. 2001, Astronomische Nachrichten, 322, 411

Nelemans, G. \& Tout, C. A. 2003, MNRAS, in preparation

Nelemans, G., Verbunt, F., Yungelson, L. R., \& Portegies Zwart, S. F. 2000, A\&A, 360, 1011

Nelemans, G., Yungelson, L. R., Portegies Zwart, S. F., \& Verbunt, F. 2001, A\&A, 365, 491 